\title{
Automatic data acquisition system for testing photovoltaic conversion chains performances in real conditions
}

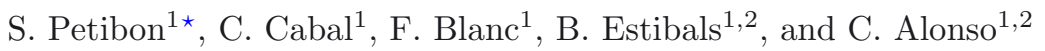 \\ 1 CNRS, LAAS, 7 avenue du Colonel Roche, 31077 Toulouse, France \\ ${ }^{2}$ Université de Toulouse, UPS, INSA, INP, ISAE, LAAS, 31077 Toulouse, France
}

Received: 4 May 2010 / Accepted: 13 September 2010

\begin{abstract}
The analyses of the performance of a complete photovoltaic system require precise measuring equipment in order to obtain reliable comparative results. This paper presents a computer-based instrumentation system suitable for a characterization of photovoltaic conversion chains. Consisting in a data acquisition stage, a data processor, photovoltaic generators and power conditioners, the system evaluates the respective efficiency of maximum power point tracker (MPPT) and power conditioner. The efficiency of unknown MPPT functions is estimated with great accuracy by means of an extremum control-based MPPT used as a reference. Efficiency evaluations of two photovoltaic battery chargers during 4 days illustrate the estimation procedure and the system test facilities.
\end{abstract}

Keywords: Data acquisition; photovoltaic system; MPPT; efficiency; converter

\section{Introduction}

Nowadays, research spends millions of Euros to increase the efficiency of photovoltaic cells of some percents. These advancements are significant only if the photovoltaic cell or photovoltaic cells groups always operate at their Maximum Power Point (MPP). Generally, a power converter associated with a function called Maximum Power Point Tracking (MPPT) is introduced between the photovoltaic module (PV) and the load, constituting a photovoltaic conversion chain, to achieve this goal. The literature proposes many MPPT strategies which guarantee to work at the maximum power point of the $I-V$ curve of the $\mathrm{PV}$ array. But, in practice there are numerous factors which cause the actual operating point to vary from the true MPP. For example, the MPPT commands that use search algorithms which oblige the operating point to oscillate around the MPP (Perturb \& Observe). These oscillations induced by a voltage or duty cycle steps [1] cause in the finite time a power transfer loss. The same goes for the MPPT command that uses the proportionality relations based on the short-circuit current $\left(I_{S C}\right)[2,3]$ and to open circuit voltage $\left(V_{O C}\right)$ [4], in order to obtain the MPP. In this case, the different parameters are specific to electrical PV characteristics and defined in the constant conditions. Thus, when atmospheric conditions variations appear (irradiance level, temperature, fluctuation, aging cells and others as we can see in Ref. [5]), these parameters values are not well adapted to localize the new MPP with accuracy. These MPPT inaccuracies conspire to reduce the

* Correspondence: spetibon@laas.fr conversion efficiency of the PV array and therefore the overall efficiency of the system. Generally, the manufacturers indicate an MPPT accuracy and efficiency value likely based on the resolution of the MPPT research algorithm or on one measure point obtained in the laboratory. The MPPT command quality evaluation under the outdoor conditions requires the knowledge of the MPP value and the operating point position according to the climatic conditions. Only short papers, like $[6,7]$ and [8], present techniques and test benches used to characterize MPPT efficiency and photovoltaic conversion chains. So the aim of our paper is to describe precisely and step by step the approach of development of a dedicated photovoltaic test bench for the community. This work performed, with consideration, will be a first step in the normalization of the efficiency characterization of the numerous conversion chains proposed nowadays on the market and by laboratories.

So in this paper, we are presenting an automatic data acquisition system dedicated for a photovoltaic application operating in real conditions. The test bench associated with a methodology of calibration allows an accurate MPP determination of several PV panels. Thus, in the same atmospheric conditions, various battery chargers associated with different MPPT methods can be compared during short (some hours) or long (days, weeks, months,... ) periods. In Section 2, the different efficiency definitions of photovoltaic chain conversion are defined. The test bench features and the measurement methodology details are illustrated in Section 3. Efficiency Evaluation of three photovoltaic battery chargers is shown in 


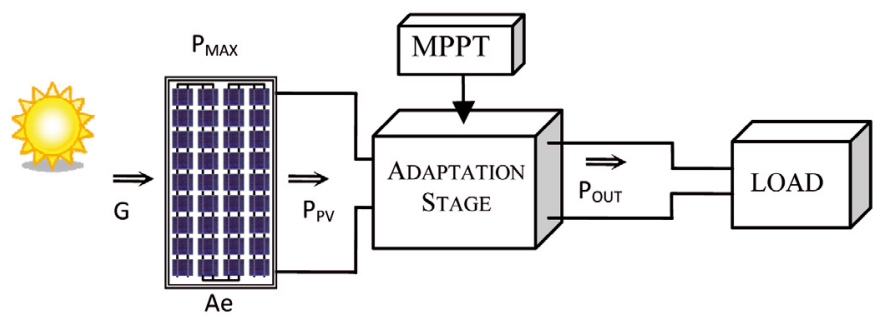

Fig. 1. Elementary photovoltaic conversion chain.

Section 4 illustrating the test bench facilities. Conclusions are contained in Section 5.

\section{Photovoltaic conversion chain}

The photovoltaic conversion chain is illustrated in Figure 1 and is generally constituted by a PV module (power source) connected to an adaptation stage associated with the MPPT command. The nature of the adaptation stage depends on the load. The grid connected applications require an inverter to transform the $\mathrm{PV}$ output continue values (DC) into alternative values (AC). When the load is a battery to accumulate the PV energy (stand alone applications), a DC-DC power converter is necessary to adapt the voltage level values between the PV output and the battery.

The different variables illustrated in Figure 1 correspond respectively to:

- $G$ : the sun irradiance $\left[\mathrm{W} / \mathrm{m}^{2}\right]$,

- Ae: the effective area of PV module $\left[\mathrm{m}^{2}\right]$,

- $P_{\mathrm{MAX}}$ : the PV maximal power point according to the temperature and the sun radiation,

- $P_{\mathrm{PV}}$ : the PV power extracted [W],

- POUT: the power transferred to the load [W].

The overall system efficiency of a photovoltaic conversion structure $\left(\eta_{\text {TOTAL }}\right)$ is given by $(1)$ :

$$
\eta_{\mathrm{TOTAL}}=\eta_{\mathrm{PV}} \eta_{\mathrm{MPPT}} \eta_{\mathrm{CONV}}
$$

where $\eta_{\mathrm{PV}}$ is the PV efficiency. The latter corresponds to the ratio of the maximum available electrical power of the panel to the entering solar irradiance by effective area.

$$
\eta_{\mathrm{PV}}=\frac{P_{\mathrm{MAX}}[\mathrm{W}]}{G\left[\mathrm{~W} / \mathrm{m}^{2}\right] A e\left[\mathrm{~m}^{2}\right]} .
$$

The quality of the MPPT commands can be evaluated by means of the MPPT efficiency $\left(\eta_{\mathrm{MPPT}}\right)$. In fact, this efficiency corresponds to the system operating-point position in respect to the available maximum power point (MPP) as illustrated in Figure 2. This efficiency is given by equation (3) which corresponds to the real available electrical power of the panel $\left(P_{\mathrm{PV}}\right)$ to its maximum available electrical power $\left(P_{\text {MAX }}\right)$.

$$
\eta_{\mathrm{MPPT}}=\frac{P_{\mathrm{PV}}[\mathrm{W}]}{P_{\mathrm{MAX}}[\mathrm{W}]} .
$$

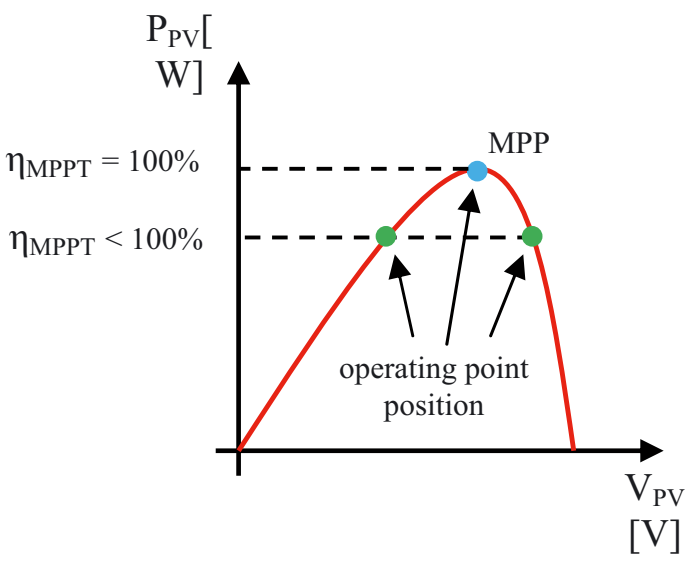

Fig. 2. Evaluation of the MPPT efficiency.

And the converter efficiency $\left(\eta_{\mathrm{CONV}}\right)$ is the ratio of the power transferred to the load $\left(P_{\text {OUT }}\right)$ of the real available electrical power of the panel $\left(P_{\mathrm{PV}}\right)$ as described by equation (4):

$$
\eta_{\mathrm{CONV}}=\frac{P_{\mathrm{OUT}}[\mathrm{W}]}{P_{\mathrm{PV}}[\mathrm{W}]} .
$$

Using equation (2) to equation (4) in equation (1), we obtain the global efficiency of the photovoltaic conversion chain:

$$
\eta_{\mathrm{TOTAL}}=\frac{P_{\mathrm{OUT}}[\mathrm{W}]}{G\left[\mathrm{~W} / \mathrm{m}^{2}\right] A e\left[\mathrm{~m}^{2}\right]} .
$$

\section{Test bench description}

\subsection{Overview}

Figure 3 shows the block diagram of the automated data acquisition system developed for photovoltaic systems characterization. Six photovoltaic conversion structures can be simultaneously analyzed. Each of them consists of a solar panel, an adaptation stage associated or not with the MPPT command and a load. Presently, the test bench is designed only to the DC-DC conversion. In this case, the adaptation stage can be battery chargers or simple dc-todc switching converters whereas the loads are basically lead acid batteries. Four variables are measured in each photovoltaic conversion chain, and sent by means of a data acquisition card to a data processing system. Namely, the photovoltaic generator voltage $V_{\mathrm{PV}}$, the photovoltaic generator current $I_{\mathrm{PV}}$, the adaptation stage output voltage $V_{\text {LOAD }}$ and the adaptation stage output current $I_{\text {LOAD }}$, as illustrated in Figure 4. Measures are recovered by means of resistive voltage dividers for the voltage and through a small resistor for the current.

For example, in our case, an adaptation stage which is placed between a group of photovoltaic cells and the battery manages the photovoltaic energy transfer thanks to the Extremum Seeking Control algorithm implemented in a microcontroller. This ESC has been chosen for this 


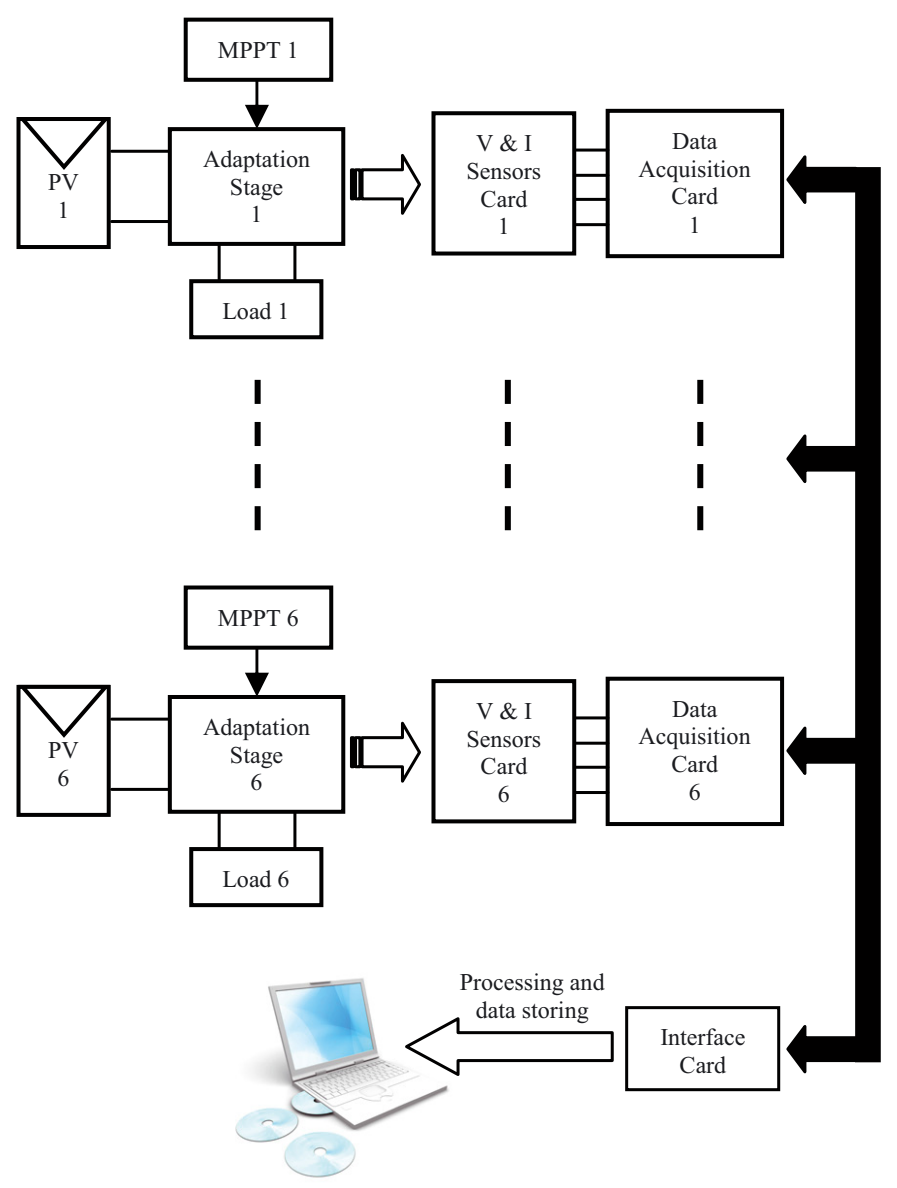

Fig. 3. Block diagram of the developed test bench.

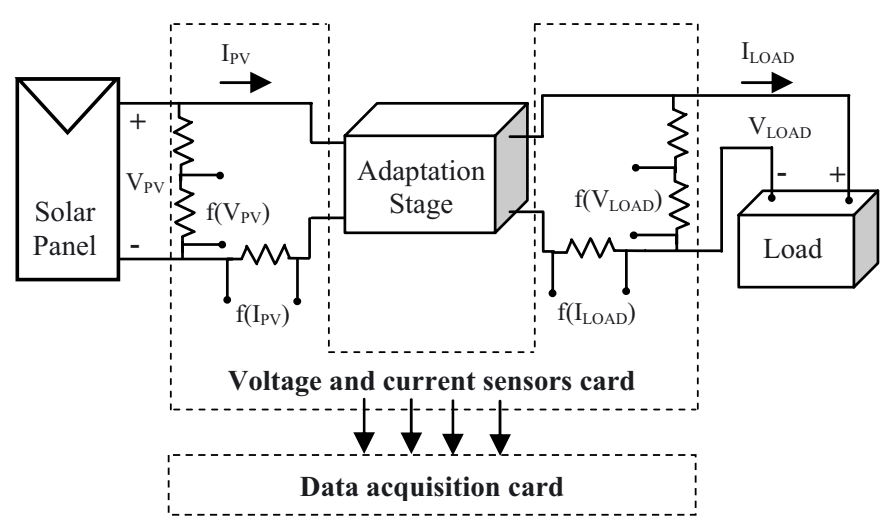

Fig. 4. Schematic representation of photovoltaic conversion chain test bench.

application for its stability, good accuracy and good Efficiency/Price ratio [9].

Experimental behavior of $I \mathrm{pv}, V \mathrm{pv}$ and $P$ pv of the PV generator with an MPPT control is shown in this section. The experimental prototype corresponds to a micro-power converter (switching frequency of $500 \mathrm{kHz}$ ) using an amorphous PV panel of 1.5 Wp from Solems (14/150/300TD). The experimental results, Figure 5 , show the steady-state behavior of the different variables at the output of the PV system with a semi-digital MPPT control [10]. The

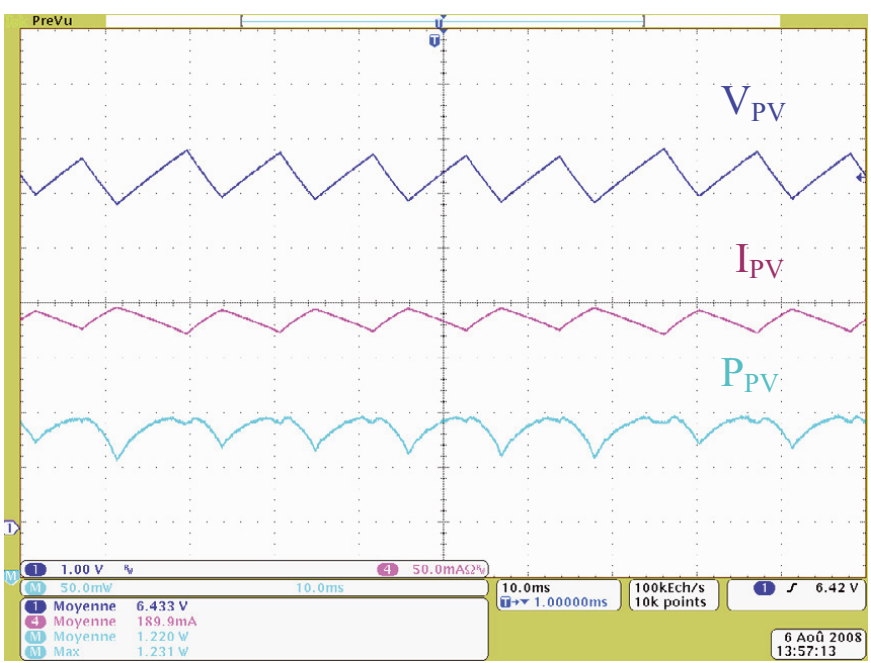

Fig. 5. Experimental steady state behavior of PV array variables controlled by a Boost with Extremum-Seeking MPPT control.

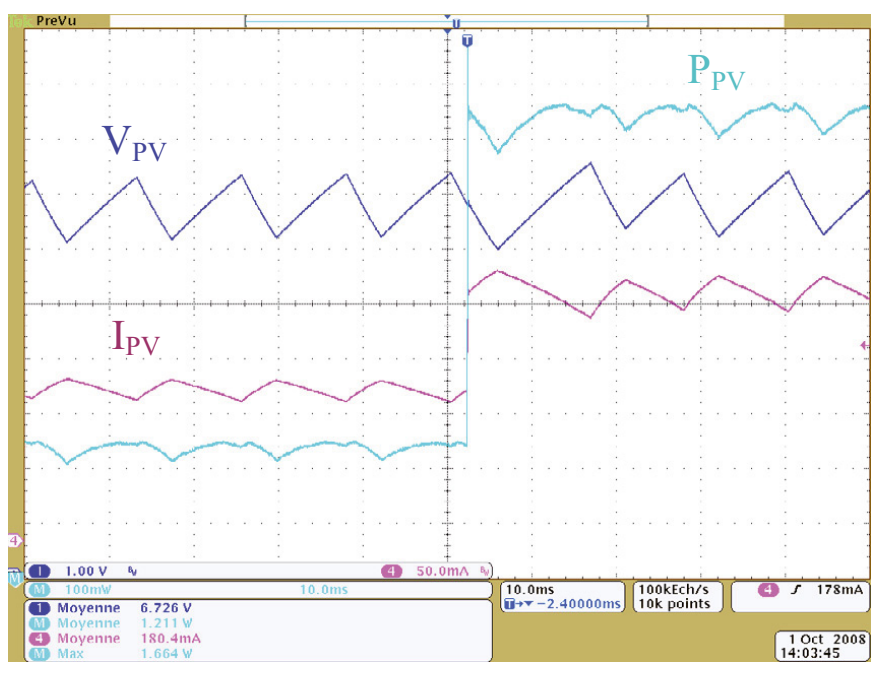

Fig. 6. Response of Boost converter with MPPT to a perturbation of irradiance.

extremum seeking control produces an oscillatory behavior around the equilibrium MPP [11]. The average value of $P \mathrm{pv}$ is $889.7 \mathrm{~mW}$ for a maximum power value of $915.5 \mathrm{~mW}$, which results in an MPPT efficiency of $97.18 \%$. Experimental results, during a rapid variation of the photovoltaic array power, are shown in Figure 6. In this case, the maximum power point has changed, and therefore, the MPPT have to adapt the system operation to the new situation. Thus, the array voltage increases linearly until reaching the new maximum power point. These results allow us to conclude that the accuracy and the dynamical behavior of the global system is quite good. As we see in this example, electrical variables measurements need good accuracy in order to get the best accuracy on power and efficiency calculation. So we have to design a dedicated photovoltaic test bench in order to ensure accurate data measurements. 


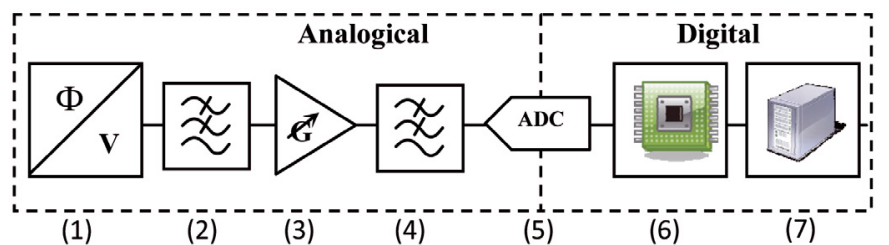

Fig. 7. Block diagram of the acquisition card.

\subsection{Acquisition card}

The acquisition chain presented in Figure 7 has the same topology for the current and voltage sensors conversion. This similitude simplifies the acquisition cards design and minimizes the delay time between each conversion chain data acquisition. The first block (1) consists of a transducer which converts the physical unit into a voltage compatible with electronic devices; simple resistive voltage divider for the voltage transducer and a resistance shunt for the current.

Resistors are specially chosen to minimize the losses in sensors and have a low drift temperature. Next, a simple RFI filter (Radio frequency interference) is inserted in order to eliminate noises from electronic power like the switching frequency of the power converter or EMI noises. Then an instrumentation amplifier INA141 in (3) offers high input impedance, assures good common-mode rejection and allows a programmable amplification of the signal. The variable gain adds the possibility to have multiple scales to increase measurement accuracy. The Butterworth filter used in (4) is one type of electronic filter design. It is designed to have a frequency response which is as flat as possible in the pass-band. The cutoff frequency of the 4th order filter is fixed at $1 \mathrm{kHz}$ to eliminate parasite frequencies which can disturb the measurement. After the level adaptation stage, an analogue to digital converter (5) samples simultaneously analogue signals $[12,13]$ and holds the result values until next sampling (at $3 \mathrm{kHz}$ sampling frequency). All measuring cards are synchronized so all samples are obtained simultaneously. The analogue signals are digitally coded by means of 16 bits. A microcontroller (6) saves and performs a preprocessing of the data described in Figure 8. This step consists first in correcting the measure function of the calibration results (see next section) and in calculating the average of the current and the voltage on 1024 points of measurements to lighten file size and finding the maximum power point (see calibration step). Data are then transferred to the computer every $340 \mathrm{~ms}\left(1024 / f_{\text {sampling }}\right)$ by means of a USB connection (7).

Data are saved in a PC file and can be displayed, in real time, on a graphic interface performed with LabWindows/CVI software. This interface allows checking the operation of each channel and seeing the evolution of the power produced by each conversion chain. In Figure 9, we can see a picture of one acquisition card used for the automatic data acquisition system. Each card has 4 connectors ( 2 inputs/2 outputs) on front panel for easier handling. Cards have been designed to be integrated in backplane

\section{Micro-Controller}

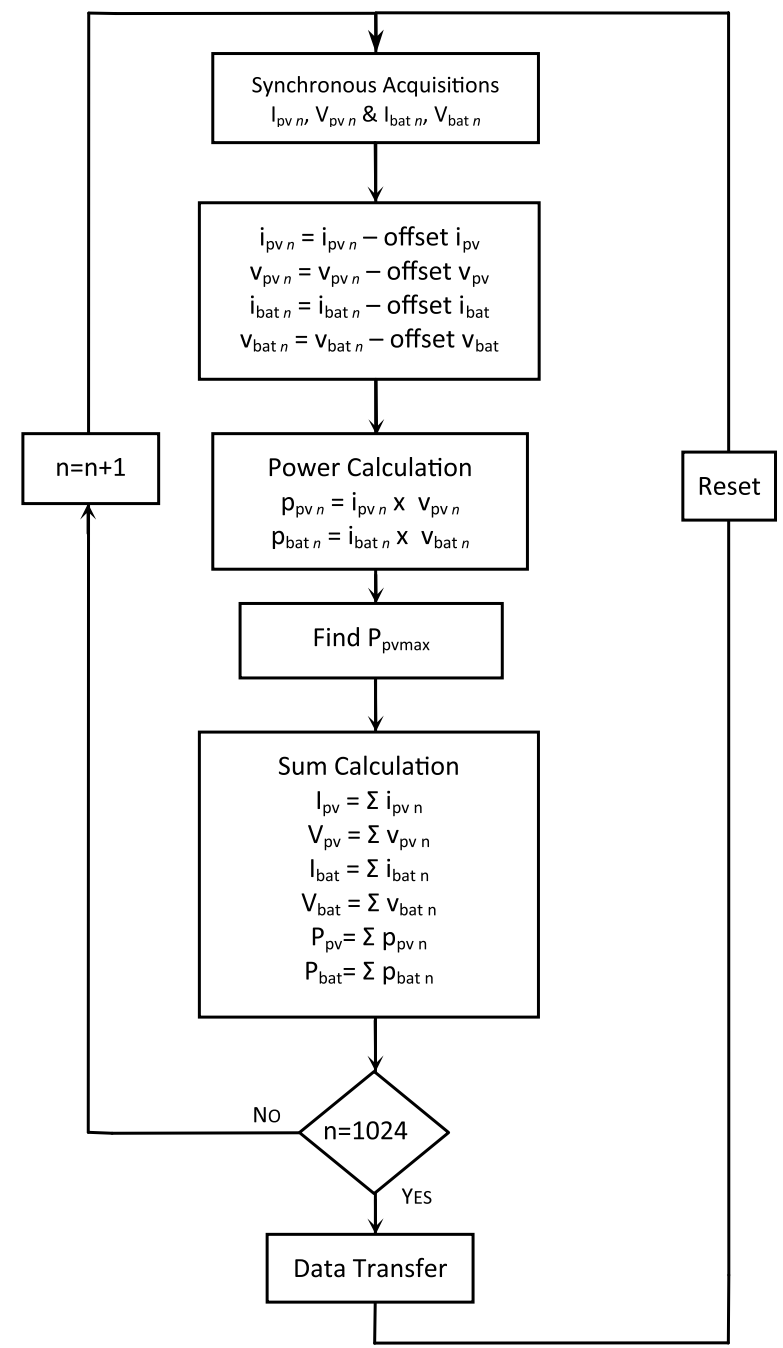

Fig. 8. Block diagram of the digital acquisition process.

by rack mount and the overall automatic data acquisition system can receive up to 6 cards. But before everything, these cards have to be correctly calibrated as we will see in the following section.

\subsection{Calibration step}

These acquisition cards must be precisely calibrated with a defined procedure to ensure the accuracy of the data measured before each test runs; therefore, we have developed an automated calibration process. Autonomous software allows a self calibration of the cards through a GPIB communication between accurate measuring devices and programmable power supply. The first step is to calibrate the current sensors. The electronic power supply is monitored to operate in current supply and zero voltage. Then, we gradually increase the current on the whole measurement scale and data are recovered (around 1000 points) 


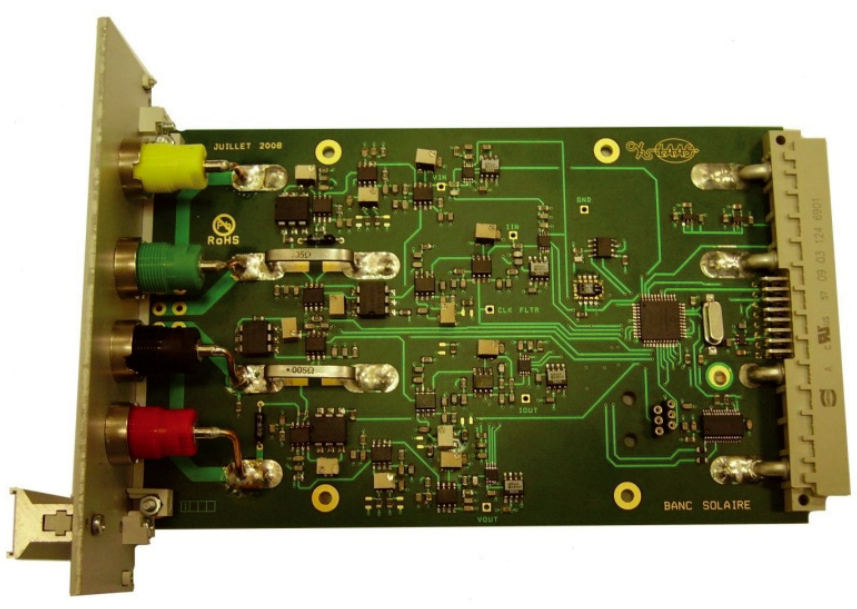

Fig. 9. Acquisition card of the photovoltaic automatic data acquisition system.

from sensor and digital multimeter (Keithley). Then we plot the reference current function of the measured current as we can see in Figure 10. A linear regression curve can easily be calculated to obtain the gain and the offset of the current sensor conversion chain. A similar approach is made to calibrate voltage sensor, but now, the electronic power supply works as a voltage source. Data are compared in order to obtain the gain and the offset of the conversion chain sensor. All data are transferred to the acquisition cards by an USB communication.

\subsection{Measurements accuracy}

At this time, five major factors of error were found. The global measurement error can be described as follows:

$$
\begin{aligned}
\varepsilon_{\text {rror }}= & \varepsilon_{\text {Offset }}+\varepsilon_{\text {Offset } t^{\circ}} \\
& +\left(\varepsilon_{\text {Gain }}+\varepsilon_{\text {Gain } t^{\circ}}\right) \times M \text { easure }+\varepsilon_{\text {Noises }} .
\end{aligned}
$$

First, the $\varepsilon_{\text {Offset }}$ is an error of offset induced by the measurement chain (amplifier, filter...). This offset is constant and can be easily compensated thanks to the calibration steps. The gain error is another type of error which is function of the measurement. In Figure 11, we can see influences of different errors on current measurements. The $\varepsilon_{\text {Gain }}$ creates an increase of the error when the currents measured increase. The last error is defined as noises error. The $\varepsilon_{\text {Noises }}$ is a set of complex errors as electronic noises, ADC resolutions and the fact that every time we repeat a measurement, we obtain slightly different results. Temperature can also disturb measurements as parameters $\varepsilon_{\text {Gain } t^{\circ}}$ and $\varepsilon_{\text {Offset }} t^{\circ}$. Tests, in controlled temperature condition (Laboratory oven), have shown a

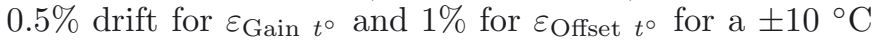
variation (the reference temperature is $25^{\circ} \mathrm{C}$ ).

The mathematical model (6) of accuracy measurements is not perfect, but it allows a global vision of the dif-
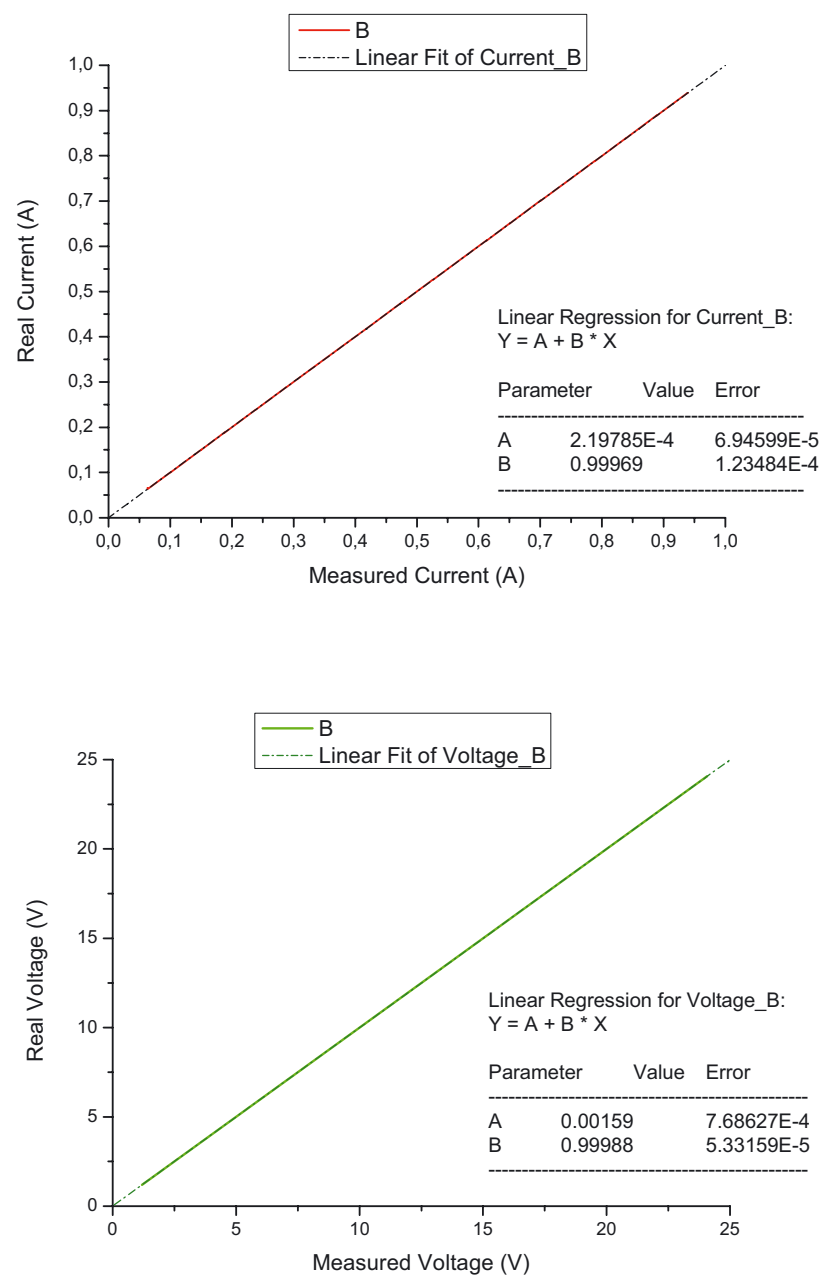

Fig. 10. Calibration curves for current and voltage sensors.

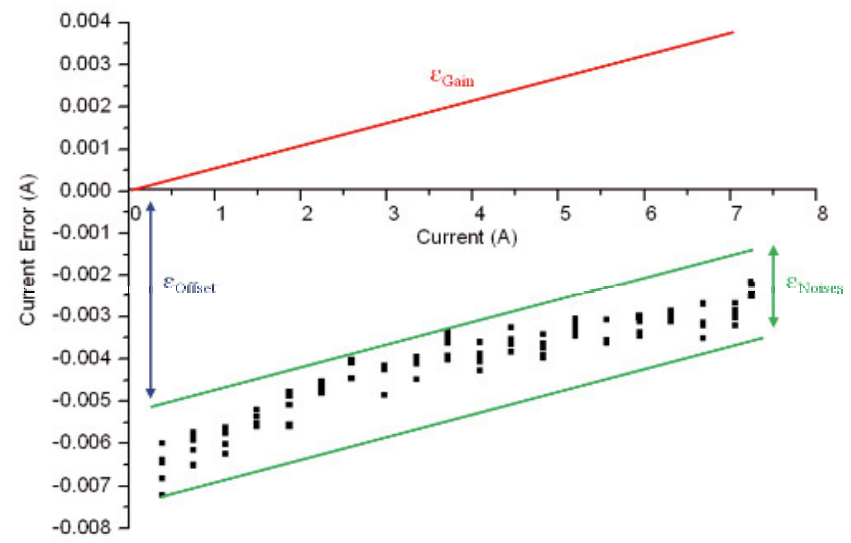

Fig. 11. Influence of Errors on current measurements.

ferent parameters which can disturb measurements. Estimating the accuracy measurement of the acquisition cards is a hard work which must be made carefully in order to obtain the most accurate measurements. As we have seen in this description, accuracy can be influenced by numerous parameters which must be defined with the intention of minimizing them. 


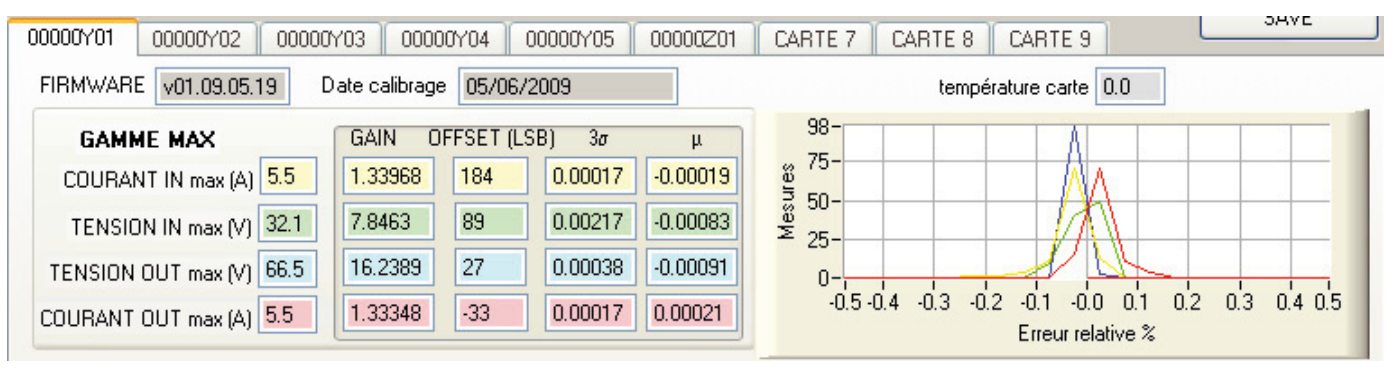

Fig. 12. Autonomous software calibration.

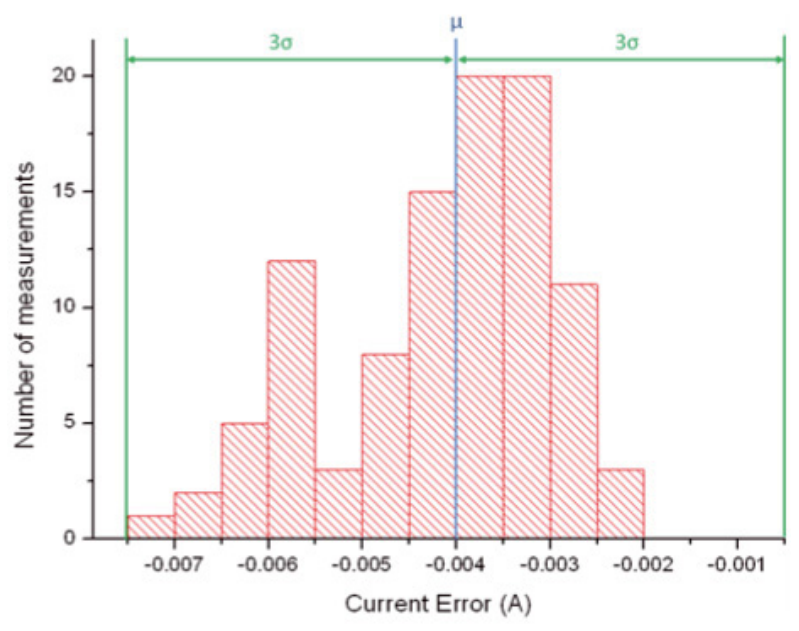

Fig. 13. Current errors bar graph.

Table 1. Measurement uncertainty for an individual acquisition card.

\begin{tabular}{lccc}
\hline Measured variable & Value & Uncertainty & Uncertainty [\%] \\
\hline$V_{\text {in }}$ & $32 \mathrm{~V}$ & $\pm 3 \mathrm{mV}$ & $\pm 0.009 \%$ \\
$I_{\text {in }}$ & $5.5 \mathrm{~A}$ & $\pm 360 \mu \mathrm{A}$ & $\pm 0.0065 \%$ \\
$V_{\text {OUT }}$ & $66 \mathrm{~V}$ & $\pm 1.29 \mathrm{mV}$ & $\pm 0.0195 \%$ \\
$I_{\text {out }}$ & $5.5 \mathrm{~A}$ & $\pm 380 \mu \mathrm{A}$ & \pm 0.0069 \\
\hline
\end{tabular}

The calibration software, in Figure 12, can also estimate the accuracy of the acquisition card. The software makes 100 measurements and calculates the relative error, difference between the card measurements and the reference, see equation (7). It gives then two parameters, $3 \sigma$ and $\mu$ for the Student's $t$-distribution law. Where $\sigma$ is the standard deviation of the error and $\mu$ is the difference between the average error and the reference. For a standard deviation of $3 \sigma, t$-distribution shows that $99.7 \%$ of the measures will be contained between $\pm 0.01 \%$ of the relative error $\left(\varepsilon_{\text {relative }}\right)$ for current and voltage measurements.

$$
\varepsilon_{\text {Relative }}=\frac{\text { Measure }- \text { reference }}{\text { reference }} .
$$

We can see in Figure 13, the $t$-distribution of the current error for 100 points of current measurements for a $5.5 \mathrm{~A}$ current. We find a deviation $3 \sigma$ equal to $170 \mu \mathrm{A}$ and a $\mu$ equal to $-190 \mu \mathrm{A}$ which means a maximum error on current measurements of $\pm 3 \sigma+\mu$, so around $360 \mu \mathrm{A}$ for high current. The uncertainty measurement process has been

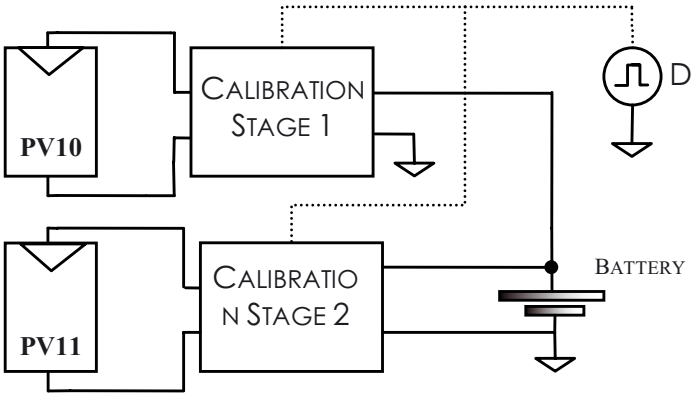

Fig. 14. Block diagram of the experimental set-up used to characterize the photovoltaic generators.

performed in accordance with the guidelines "A guide to the expression of uncertainty in measurement" with the Type A evaluation of standard uncertainty reported in [14]. Table 1 illustrates the measurement uncertainty for one acquisition card. Thanks to these rigorous approaches of calibration, we can ensure an uncertainty of $\pm 0.0159 \%$ on measured powers for the full scale range at $25{ }^{\circ} \mathrm{C}$.

\section{Comparative study}

\subsection{Panel calibration}

In order to compare the efficiencies of different conversion systems on an equal basis, we have to guarantee identical energy sources at the input. Although, electrical characteristics should be theoretically identical for panels of same size and manufacturer, in practical applications, differences in electrical performance are often found, mainly due to random scattering in the manufacturing process. Therefore, calibrating the panels is mandatory in order to evaluate their differences in the photovoltaic conversion. The calibration process, based on [15], consists in obtaining the $i-v$ characteristic of each panel on one day of measurement.

In the first step, panels 10 and 11 are connected as shown in Figure 14. Duty cycle of each DC-DC converter of calibration stage is simultaneously changed from $10 \%$ to $90 \%$ in order to display the current versus voltage characteristics of each panel. The next step, in the calibration process, is the representation of the power delivered by one system as a function of the power given by another system taken as a reference. Figure 15 shows the maximum power 


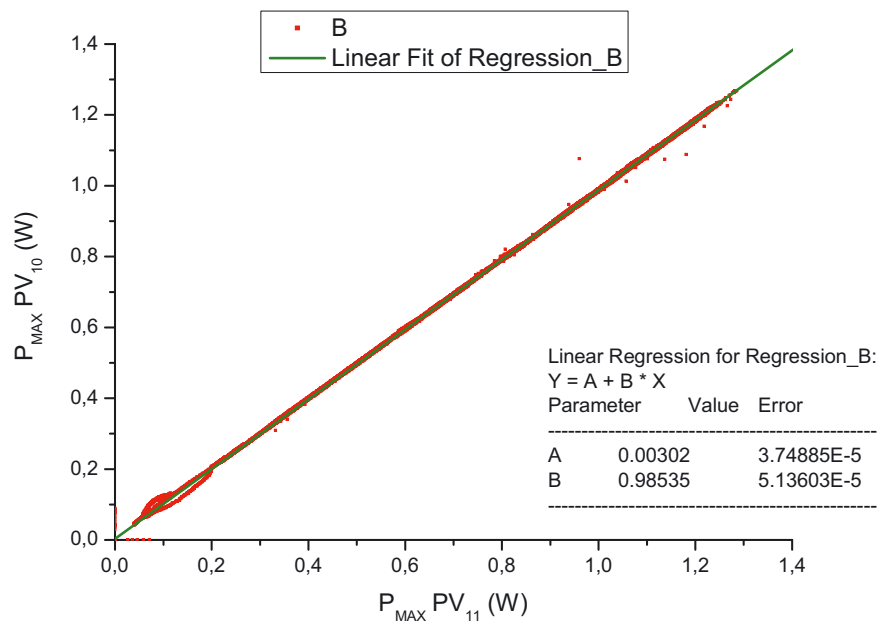

Fig. 15. Maximum output power of panel 10 as a function of the power of panel 11 .

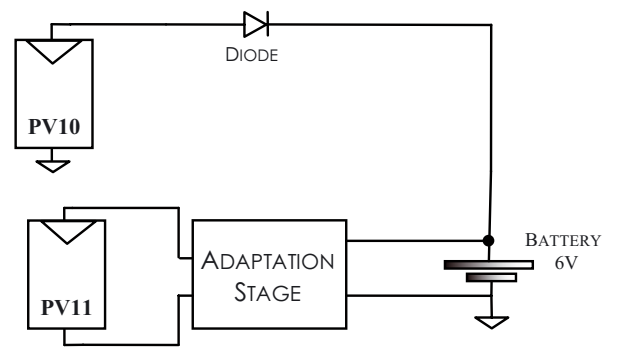

Fig. 16. Block diagram of the experimental set-up used to characterize the photovoltaic conversion chains.

delivered by the PV10 as a function of the power given by the reference PV11.

The resulting characteristic is roughly a straight line. We can approximate the representation of Figure 15 by the analytical expression:

$$
P_{\mathrm{MAX}-10}=A+B P_{\mathrm{MAX}-11} .
$$

Where $P_{\mathrm{MAX}-10}$ is defined as the estimated maximum power of PV10, $P_{\mathrm{MAX}-11}$ is the maximum power of reference measured on panel 11 and coefficients $A$ and $B$ are found by a regression procedure, their respective values being $A=0.00302$ and $B=0.98535$.

\subsection{Low-power applications}

We have initially developed the test bench for low power applications in the context of an ANR named ATOS. We have designed low power DC-DC converter with MPPT dedicated to amorphous silicon PV modules of 1 to $2 \mathrm{Wp}$. The aim is to display the benefit to insert an adaptation stage between the PV and the load compare to a direct connection for low power applications. As we can see in Figure 16, the two conversion chains are connected in parallel at the output to a same battery of $6 \mathrm{~V}$. The PV10 is directly connected to the load through a simple diode and the PV11 through an adaptation stage especially developed for this project.

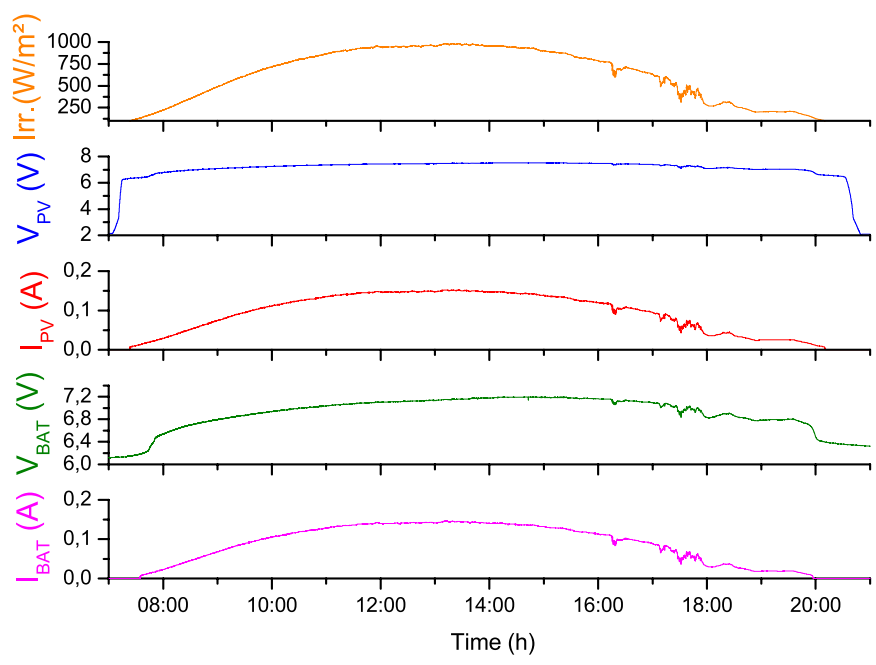

Fig. 17. Irradiance, voltages and currents input/output of the adaptation stage for one day test.

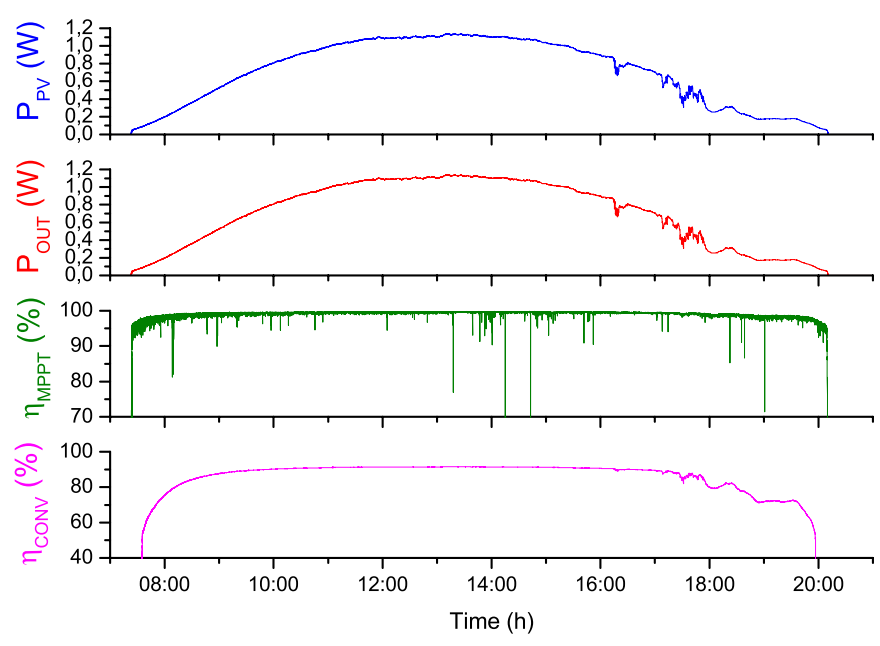

Fig. 18. Powers and efficiencies of the adaptation stage for one day test.

Figure 17 illustrates, on one operating day, the results of measurements of the photovoltaic conversion chain with the adaptation stage. The test bench allows us to recover input and output variables of the chain like the PV voltage and current $\left(V_{\mathrm{PV}}, I_{\mathrm{PV}}\right)$ and output voltage and current $\left(V_{\mathrm{BAT}}, I_{\mathrm{BAT}}\right)$.

This campaign of measurements has been done on a beautiful day of August in Toulouse, as we can see on the graph. The PV voltage (at the MPP) does not vary much, from 6.5 to $7 \mathrm{~V}$. On the other hand, the current produced by the module increases until $1 \mathrm{pm}$ to gradually decrease at the end of the day. The output voltage of the adaptation stage increases due to the state of charge of the battery. And finally, the current supply to the battery has the same shape as the PV current at the converter efficiency.

After test bench data processing, we can put up results in Figure 18. In this section, we can use the data to characterize the conversion chain on one operating day. We recover the power supply by the module $\left(P_{\mathrm{PV}}\right)$, and 


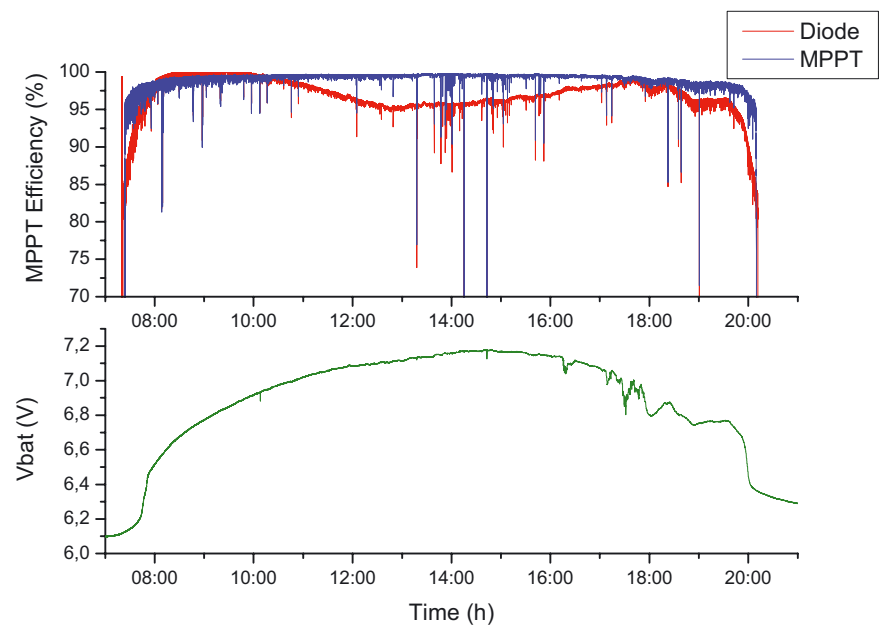

Fig. 19. Comparison of MPPT efficiencies of the two conversion chains on one day test.

the power supply to the battery $\left(P_{\text {OUT }}\right)$. And finally, we can deduce the efficiency of the MPPT $\left(\eta_{\mathrm{MPPT}}\right)$ and the efficiency of the adaptation stage $\left(\eta_{\mathrm{CONV}}\right)$. We take the micro-converter as a reference because it uses a MPPT with extremum seeking control. This type of control guarantees the attainability of the maximum power point from any initial condition and from any solar irradiation level.

Figure 19 illustrates the MPPT efficiencies of the two conversion chains. For the micro-converter with MPPT control, the $\eta_{\text {MPPT }}$ is always between $98 \%$ and $99 \%$ for the whole day. At the opposite, for the direct connection with the diode, the $\eta_{\text {MPPT }}$ value changes function of the battery charge. In the morning, the optimum voltage of the PV corresponds with battery voltage and at mid-day, $V_{\text {bat }}$ gets away from MPP and so, the MPPT efficiency decreases.

Table 2 presents a summary of the comparative measures made between a direct connection and a connection via a Boost micro-converter over one operating day. We can find in this table the maximum energy potentially available on the photovoltaic generator. We then note the real energy produced and the energy transferred to the load. With these measures, we have associated energy efficiency such as $\eta_{\text {MPPT }}$ performance, conversion efficiency $\eta_{\text {conv }}$ and the total photovoltaic conversion chain efficiency over one day.

We can observe that the $\eta_{\mathrm{MPPT}}$ is above $99 \%$ for the micro-converter and $97 \%$ for the direct connection thanks to the stability of the optimum voltage of the amorphous photovoltaic array. In spite of a good $\eta_{\text {conv }}$ for the prototype of micro-converter, the total efficiency is less important than the direct connection.

This new test bench allowed us to compare different photovoltaic connections under same outdoor conditions. First tests have proved that the micro-converter is not competitive enough to compete with the direct connection when the optimum voltage of the PV array corresponds to the voltage of the battery. However, this perfect adaptation is not a frequent configuration in one operating year.
The optimum voltage and current are functions of several parameters as irradiance, temperature, aging cells, shading effects which make the photovoltaic module an unstable source. So, adaptation stage is essential in this kind of application for a constant maximization of the PV power. Furthermore, the micro-converter includes a battery management algorithm which secures battery system and improves battery life time.

\subsection{Daily tests}

This new test bench allows us to perform the characterization of several conversion chains on several days thanks to the automatic data acquisition process as we can see in Figure 20 for four operating days. In this campaign of measurements, we have compared the same topology as present above, a direct connection and a connection with an adaptation stage connected to the same $8 \mathrm{~V}$ battery. The acquisition process runs from 7.30 am to $9.00 \mathrm{pm}$ in order to avoid unnecessary data acquisitions. Figure 20 shows electric power extracted from photovoltaic modules by each architecture. Thanks to this simple graph we can rapidly estimate the benefit of one architecture versus another one. In our case, we can see that the micro-converter with MPPT control produces more power than the direct connection in the same conditions of irradiance. The micro-converter works here always at the Maximum Power Point of the photovoltaic module. This maximization of power induces a benefit of more than $50 \%$ of photovoltaic energy on these four operating days compared with a classic direct connection.

\section{Conclusion}

The main characteristics of an automatic measurement bench for testing photovoltaic systems have been reported in this paper. A step by step strategy development has been described with a particular attention for the accuracy of measurements. Limitations in the bandwidth and noise measurements problems have been solved. We have validated the good accuracy of the bench thanks to an accurate calibration step. Finally, experimental tests have been done showing the different architectures of power management evaluations on four days. The automatic measurement system presented in this paper guarantees an objective test for the efficiency evaluation of any photovoltaic DC-DC conversion system.

So far, two test benches have been developed by our Laboratory, one first presented in this paper for low PV power applications and a second one for middle PV power (around $200 \mathrm{Wc}$ by acquisition cards). Our future works are orientated towards communication with a meteorological bench in order to correlate experimental results with irradiance, temperature, anemometer and hydrometric measurements.

Acknowledgements. Authors would like to acknowledge the financial support of the French National Research Agency for 
Table 2. Results of the two conversion stages performance on one operating day.

\begin{tabular}{|c|c|c|c|c|c|c|}
\hline & $\begin{array}{c}\text { PV available energy } \\
(\text { (W.h) }\end{array}$ & $\begin{array}{c}\text { Produced energy } \\
(\text { (W.h) }\end{array}$ & $\begin{array}{c}\eta_{\mathrm{MPPT}} \\
(\%)\end{array}$ & $\begin{array}{c}\text { Transferred energy } \\
\text { (W.h) }\end{array}$ & $\begin{array}{c}\eta_{\mathrm{CONV}} \\
(\%)\end{array}$ & $\begin{array}{c}\eta_{\text {Total }} \\
(\%)\end{array}$ \\
\hline Micro-converter & 8.727 & 8.667 & 99.23 & 7.834 & 90.38 & 89.8 \\
\hline Diode & 8.6638 (estimated) & 8.412 & 97 & 8.055 & 96 & 93 \\
\hline
\end{tabular}

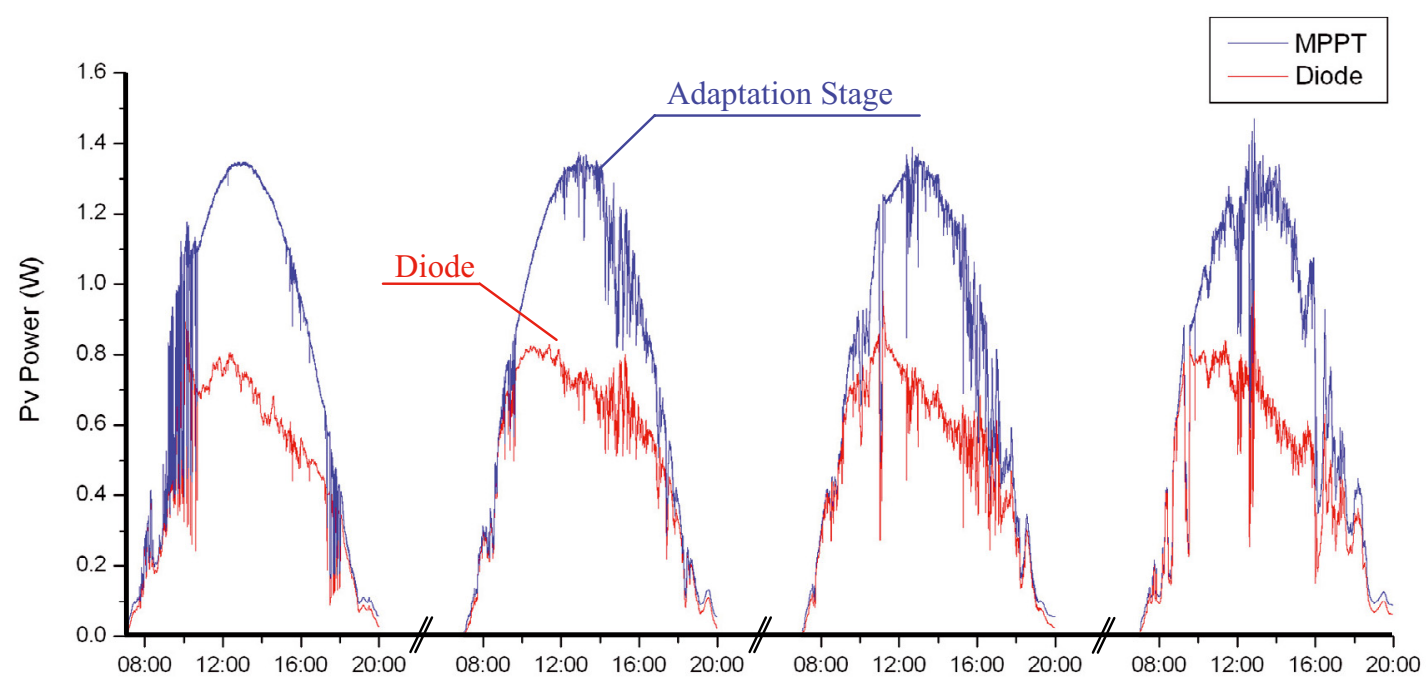

Fig. 20. Four day PV power acquisitions for 2 different architectures of power management systems.

ATOS project. And, thanks to SOLEMS for their thin-film silicon photovoltaic modules.

\section{References}

1. N. Femia, G. Petrone, G. Spagnuolo, M. Vitelli, Optimization of Perturb and Observe Maximum Power Point Tracking Method, IEEE Trans. Power Electron. 20, 16-19 (2004)

2. A.S. Masoum, H. Dehbonei, E.F. Fuchs, Theoretical and Experimental Analyses of Photovoltaic Systems with voltage and current based maximum power point tracking, IEEE Trans. Energy Convers. 17, 514-522 (2002)

3. N. Toshihiko, S. Togashi, N. Ryo, Short-current Pulse based Maximum Power Point Tracking Method for Multiple Photovoltaic-and-Converter Module System, IEEE Trans. Ind. Electron. 49, 217-223 (2002)

4. J. Ghaisari, M. Habibi, A. Bakhshai, An MPPT controller design for photovoltaic (PV) system based on the optimal voltage factor tracking, in IEEE Electr. Power Conf. (2007), pp. 359-362

5. R. Laronde, A. Charki, D. Bigaud, Reliability of photovoltaic modules based on climatic measurement data, Int. J. Metrol. Qual. Eng. 1, 45-49 (2010)

6. M. Benghanem, A. Maafi, Data acquisition system for photovoltaic systems performance monitoring, IEEE Trans. Instrum. Meas. 47, 30-33 (1998)

7. Z. Prochazka, J. Machacek, System for processing, vizualizaition and data storage, in Int. Conf. on Modern Technique and Technologies (MTT), 2008, pp. 41-44
8. M. Jantsch, M. Real et al., Measurement of PV maximum power point tracking performance, in 14th European Photovoltaic Solar Energy Conf., Barcelona, 1997

9. R. Faranda, S. Leva, Energy comparison of MPPT techniques for PV Systems, WSEAS Trans. Power Syst. 3, 446 (2008)

10. S. Petibon, C. Alonso, B. Estibals, L. Seguier, C. Cabal, P. Roca i Cabarrocas, E.V. Johnson, A. Abramov, New distributed architecture for Tandem solar cells based on pm$\mathrm{Si}: \mathrm{H} / \mu \mathrm{c}-\mathrm{Si}: \mathrm{H}$ structures, in IEEE Int. Symp. on Industrial Electronics (ISIE), Cambridge, UK, 2008, pp. 1542-1547

11. R. Leyva, I. Queinnec, C. Alonso, A. Cid-Pastor, D. Lagrange, L. Martinez-Salamero, MPPT of photovoltaic systems using extremum seeking control, IEEE Trans. Aerosp. Electron. Syst. 42, 249-258 (2006)

12. B. Baker, A glossary of Analog to digital specifications and performance characteristics, sbaa147a, Texas Instruments Incoporated (2008)

13. Burr-Brown, Analog to digital converter grounding practices affect system performances, sbaa052, Application Bulletin (2000)

14. International Bureau of Weights and Measures, Evaluation of measurement data- Guide to the expression of uncertainty in measurement, JCGM 100 (2008)

15. A. Cid-Pastor, C. Alonso, B. Estibals, D. Lagrange, L. Martinez-Salamero, Automatic measurement system for testing photovoltaic conversion chains, in 30th Annual Conf. IEEE Industrial Electronics Society (IECON), 2004, Vol. 3, pp. 3076-3081 